\title{
Care pathways in thrombosis management: the INNOVATE peer-to-peer educational initiative
}

This article was published in the following Dove Press journal:

Advances in Medical Education and Practice

6 March 2017

Number of times this article has been viewed

\author{
Raj K Patel \\ King's College Hospital, London, UK
}

Correspondence: Raj K Patel Department of Haematological Medicine, King's Thrombosis Centre, 4th Floor Hambleden Wing West, King's College Hospital, Denmark Hill, London, SE5 9RS, UK

Tel +442032994152

Fax +44 20 8I8I 47|I

Email rajpatel@nhs.net
Context: Anticoagulant options for the management of venous thromboembolism (VTE) now include the non-vitamin $\mathrm{K}$ antagonist oral anticoagulants (NOACs). The safe and effective integration of these agents into routine clinical practice within different health care settings presents common challenges. Bayer AG created the INternational Network fOr Venous and Arterial Thrombosis Excellence in practice (INNOVATE) program as a professional education network to foster best practice in thrombosis management in the NOAC era.

Concept and format: Since 2013, INNOVATE has been run as a series of educational 1.5-day global meetings at VTE centers of excellence. The format is based on expert-led discussion rather than lectures; all participants are encouraged to share their own expertise and experience. Through peer-to-peer exchange, less experienced professionals from an array of specialties learn from others in a small-group interactive setting. This format encourages positive engagement and discussion, and the establishment of relationships between health care professionals from different countries.

Expansion and localization: INNOVATE has successfully expanded to cover a broad spectrum of thromboembolic disorders in which anticoagulation with NOACs plays an important role; now including specific meetings focusing on the management of patients in the community. Local meetings are run in many countries in the local language, facilitating discussion and ensuring applicability to local or regional issues.

Implementing learnings: INNOVATE delegates have provided consistently positive feedback and have used their attendance to create and improve thrombosis management pathways in their own institutions. Overseen by a Steering Committee, the program responds to feedback, evolving to meet the needs of participants.

Conclusion: By showcasing best practice in the care of patients requiring anticoagulation, INNOVATE provides a model for the non-promotional support of medical education by industry. The objectives are to encourage the responsible use of new drugs (specifically NOACs) and their integration into existing and new care pathways and to ensure that every patient receives the benefit of evidence-based clinical practice.

Keywords: professional education, venous thromboembolism, anticoagulation, cardiovascular disease, multidisciplinary health care

\section{Introduction}

Pharmaceutical companies commonly sponsor educational programs around their products. This practice has been recognized by industry guidelines and health care service providers as important for educating health care professionals on new drugs, with the stipulation that comprehensive, balanced, and unbiased information must be 
provided. ${ }^{1,2}$ For this reason, the sponsorship of educational events by industry through unrestricted educational grants in which an independent faculty of experts is responsible for the content and delivery of the event - is seen as the most appropriate approach.

Thromboembolic disorders are a recognized global health burden: thrombosis is responsible for one in four deaths worldwide, and the incidence is increasing because of aging populations. ${ }^{3}$ Anticoagulant options for the management of thromboembolic disorders have been enhanced in recent years by the approvals of four non-vitamin $\mathrm{K}$ antagonist (VKA) oral anticoagulants (NOACs) for the prevention of stroke in patients with non-valvular atrial fibrillation (NVAF), the primary prevention of venous thromboembolism (VTE) after major orthopedic surgery, and the acute treatment and secondary prevention of VTE. ${ }^{4-7}$ These NOACs - the direct thrombin inhibitor dabigatran and the direct Factor Xa inhibitors apixaban, edoxaban, and rivaroxaban - have become increasingly used in these settings because, unlike VKAs such as warfarin, they have predictable pharmacokinetic and pharmacodynamic properties that allow for fixed dosing across broad patient populations without the need for routine coagulation monitoring. They also have fewer drug-drug interactions and diet and lifestyle restrictions for patients. ${ }^{8}$

Since its initial approval in 2008, rivaroxaban has become the most-used NOAC worldwide, with $>18$ million patients treated (Bayer AG, unpublished data). As well as the previously mentioned indications, rivaroxaban is also approved in Europe for secondary prevention of recurrent ischemia in stabilized patients with elevated cardiac biomarkers after an acute coronary syndrome event. ${ }^{4}$ Bayer AG (Berlin, Germany), the manufacturer of rivaroxaban, has put in place a "responsible use program" to support the appropriate prescribing of rivaroxaban across these diverse settings.

Multiple challenges in integrating NOACs into clinical practice have been encountered (Figure 1). Large volumes of clinical trial data were released in a short space of time, and different NOACs with different mechanisms of action and dosing requirements became available, creating the potential for confusion in a field where VKAs had been the only oral anticoagulant option for half a century. Many academic societies publish practical guidelines for physicians, but the need to gain initial experience with the NOACs led to a lag in the updating of these recommendations. A further challenge was the lack of standardization in the management of VTE (and indeed other thromboembolic disorders), with no single discipline having overall responsibility for anticoagulant prescribing in many health care settings. ${ }^{9}$ There was, therefore, a clear need to provide education to a multidisciplinary cross-section of health care professionals around the use of NOACs and their integration into existing protocols and pathways. The relative convenience of the NOACs compared with VKAs also represented an opportunity to streamline and refine patient pathways in thrombosis management for the benefit of both patients and clinicians.

In late 2012, representatives of Bayer undertook discussions with leading health care professionals who were involved in the multidisciplinary management of pulmonary

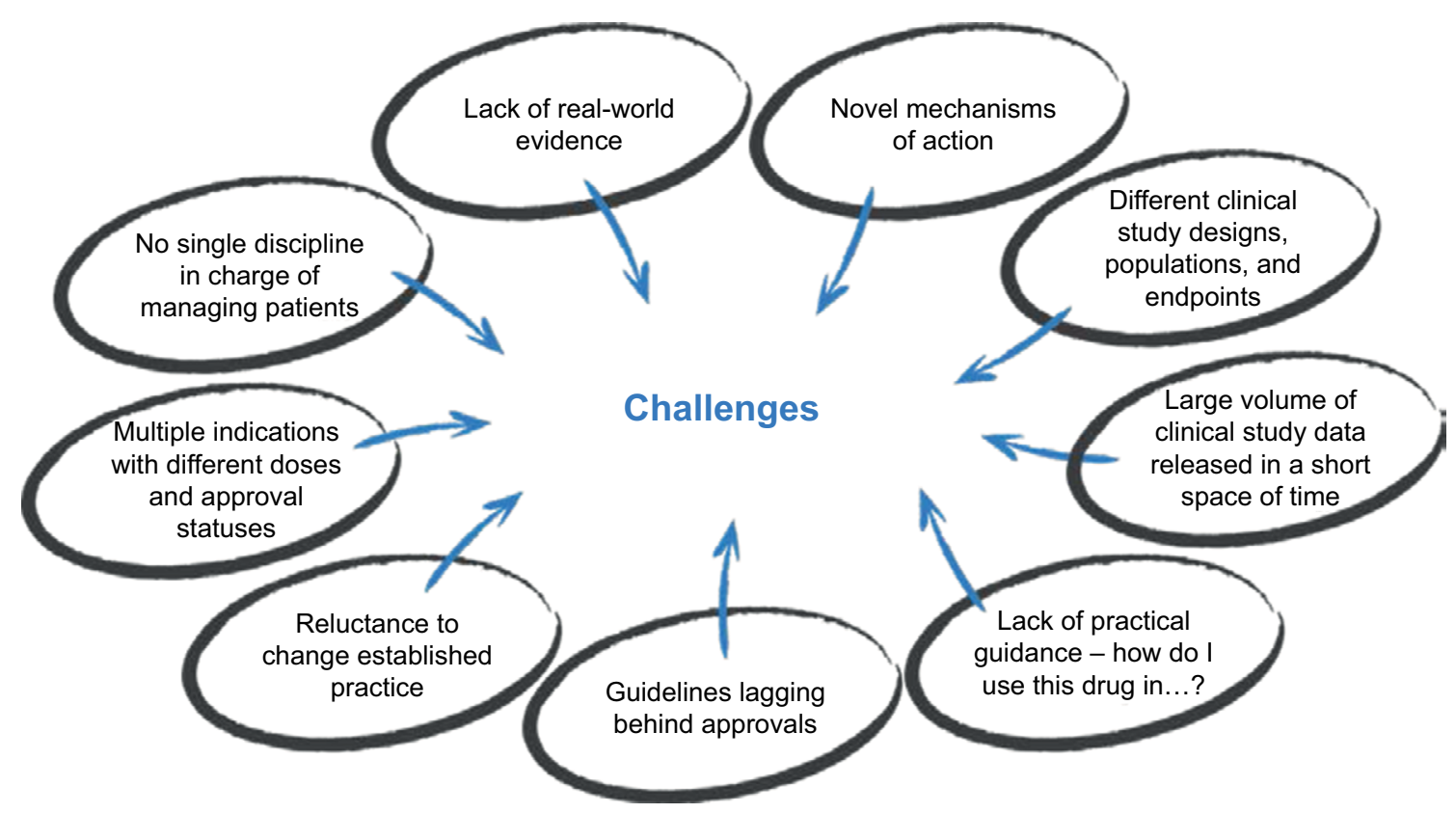

Figure I Challenges in integrating non-vitamin $\mathrm{K}$ antagonist oral anticoagulants into clinical practice. 
embolism (PE) and deep vein thrombosis (DVT) in the UK to identify the educational needs and practical support required to facilitate the responsible use of rivaroxaban and the NOACs more widely. The result was the inception of the educational peer-to-peer INternational Network fOr Venous and Arterial Thrombosis Excellence in practice (INNOVATE) program, a partnership that was initially formed between Bayer and thrombosis experts at two UK centers of excellence: King's College Hospital NHS Trust, London and the Royal Hallamshire Hospital, Sheffield. From an initial meeting in Sheffield in September 2013, the INNOVATE program has expanded globally to the extent that $>700$ health care professionals have now attended one of the 23 global INNOVATE meetings that have taken place in three countries (the UK, Germany, and Canada) to the end of 2015, with many more attending local events. This review article describes the conduct of, and learnings from, this exemplar program in peer-to-peer information exchange and education.

\section{The concept of INNOVATE}

The core principle of the INNOVATE program is that every patient should receive the benefit of consistent, evidencebased clinical practice with the aim of improving outcomes in thromboembolic disease. The INNOVATE faculty members seek to support participants to effect change and achieve best-practice outcomes for every patient, and strive to provide participants with the tools to effect change and improve the management of thromboembolic disorders in their daily practice. This is very different from the replication of one center's pathway by another, but rather involves taking relevant best-practice examples and clinical information and fitting these to the reality of a different health care system.
Through peer-to-peer exchange, professionals who are less experienced in the initiation or management of anticoagulants can learn from others in a small-group setting that maximizes interactivity. Shared tools and guidance, including pathways and protocols, give clinicians a head start in supporting change and in achieving best practice.

King's College Hospital NHS Trust, London, and the Royal Hallamshire Hospital, Sheffield, were selected as the initial hosts for the INNOVATE meetings because, as well as being centers of excellence in VTE management, they exemplify a multidisciplinary approach to patient care using validated patient pathways to deliver a consistent and integrated service. The committed expert faculty reflects the multidisciplinary approach to VTE care and not only includes hematologists and respiratory physicians but also reflects the innovative use of other health care providers, such as specialist nurses and pharmacists in these centers. In particular, both centers have pioneered the use of a nurse-led DVT outpatient service, which not only helped to standardize the quality of patient care and outcomes but also provides economic benefits and facilitates the professional advancement of nursing staff. Similar opportunities for specialism have also arisen for pharmacists and allied health care service personnel. Each specialist brings their own set of objectives and complementary skills to the management of patients with VTE (Table 1).

\section{Format of the global INNOVATE program}

INNOVATE is centered on a series of global 1.5-day meetings for small groups of international participants ( $\sim 25$ individuals per meeting), who are encouraged to network, share their

Table I Objectives and core skills of the members of the multidisciplinary VTE management team at King's College Hospital

\begin{tabular}{|c|c|c|}
\hline Multidisciplinary team members & Objectives in managing VTE & Core skills and knowledge \\
\hline Hematologist & $\begin{array}{l}\text { - Develop diagnostic pathways for VTE } \\
\text { - Provide appropriate anticoagulant therapy } \\
\text { - Manage bleeding risk and treatment duration } \\
\text { - Prevent secondary complications }\end{array}$ & $\begin{array}{l}\text { - Specialist knowledge of (anti)coagulation and } \\
\text { thrombosis } \\
\text { - Overview of latest clinical data on anticoagulant } \\
\text { therapy }\end{array}$ \\
\hline Pulmonologist & $\begin{array}{l}\text { - Risk-stratify patients with PE } \\
\text { - Prevent secondary complications }\end{array}$ & - Expertise in the diagnosis and management of PE \\
\hline DVT nurse & $\begin{array}{l}\text { - Manage patients with DVT according to } \\
\text { established clinical standards and protocols }\end{array}$ & $\begin{array}{l}\text { - Protocol-driven consistency of approach } \\
\text { - Holistic approach to patient care and education }\end{array}$ \\
\hline Pharmacist & $\begin{array}{l}\text { - Ensure correct dosing of anticoagulants } \\
\text { - Measure drug levels when needed } \\
\text { - Avoid drug interactions }\end{array}$ & $\begin{array}{l}\text { - Comprehensive knowledge of drug licenses, } \\
\text { dosing, drug interactions, and contraindications }\end{array}$ \\
\hline Vascular surgeon & $\begin{array}{l}\text { - Provide surgical intervention for treating VTE, } \\
\text { where appropriate }\end{array}$ & $\begin{array}{l}\text { - Specialized in techniques such as thrombolytic } \\
\text { therapy and surgical embolectomy }\end{array}$ \\
\hline Diagnostician & - Confirm diagnosis of VTE & $\begin{array}{l}\text { - Expertise in diagnostic scanning and interpretation } \\
\text { of results }\end{array}$ \\
\hline
\end{tabular}

Abbreviations: DVT, deep vein thrombosis; PE, pulmonary embolism; VTE, venous thromboembolism. 
experiences, and maintain contact with the faculty after the event. The criteria for participation are broad, but individuals should be involved in the hands-on delivery of thrombosis care and may come from an array of specialties and backgrounds. Indeed, the more diverse the group, the wider the range of experiences shared and the more engaging, interactive, and useful the meeting generally proves to be.

The format of the INNOVATE meetings is fairly consistent, although the content varies depending on the approach and specialties of each center. The flow of the agenda broadly follows the patient flow through the King's College Hospital patient pathways (Figure S1), with presentations by individuals representing each group in the multidisciplinary team. Presentations are based on each health care professional's own daily practice and expertise and are interactive rather than didactic, often using case studies to provide a real-world emphasis. The presentation of large volumes of clinical data is generally avoided, but pertinent data are used to illustrate the rationale for particular approaches to patient management. All meetings have a practical focus centered on active involvement by participants and a similar weight is given to time for presentation and for discussion. To provide further motivation for attendance at the meetings, the UK events are accredited with continuing professional development (CPD) points.

Over time, the content of the INNOVATE meetings has changed to reflect a growing familiarity with the NOACs within the health care community. Initially, content was focused on basic questions regarding dosing, contraindications, use in special populations, etc. Although the basics remain, the focus has shifted to the practical challenges of turning national clinical guidelines into patient pathways within the hospital and wider community care structure in the NOAC era, as well as focusing on pragmatic approaches to diagnostics and treatment in areas for which published data are limited. Several former participants (known as INNOVATE Alumni) have used their attendance at an INNOVATE meeting to improve care pathways within their own institutions and local areas (discussed in the "Implementing learnings from INNOVATE in local VTE protocols" section), and some of these individuals have subsequently returned to participate in other meetings to showcase their achievements, thereby motivating others to effect similar changes. In this way, the program continues to constantly respond to the needs of the thrombosis community.

Time is built into the schedule of an INNOVATE meeting to allow participants to liaise with each other and discuss specific issues in more depth on an ad hoc basis. The value of INNOVATE as a networking tool has been demonstrated in my institution by the formation of an ongoing link between
King's College Hospital and hospitals in Portugal. This came about as a result of the attendance of a number of Portuguese physicians at the INNOVATE meeting in London. Since then, we have been receiving a steady stream of junior physicians from Portugal, who spend a 3-month period at King's to learn about VTE management. A number of our visiting Portuguese interns have undertaken scientific projects at King's, resulting in presentations at national and international conferences and a research publication. ${ }^{10}$

\section{Expansion and localization of INNOVATE Global expansion}

In 2014, the global INNOVATE program expanded to encompass further centers of excellence in Ontario, Canada (McMaster University) and Mainz, Germany (Center for Thrombosis and Haemostasis, University of Mainz). Meetings at the latter center are conducted in German. These meetings include a focus on the prevention of stroke in patients with NVAF, as well as the prevention and treatment of VTE, and, therefore, also attract cardiologists and specialist cardiology nurses. To provide oversight and to share best practice, the Chairs of the global meetings meet regularly as a Steering Committee to define the ways in which the program should evolve. This has included two international meetings for cardiologists to specifically cover NVAF and acute coronary syndromes, respectively, both of which were held in 2015. By responding to educational needs in this way, INNOVATE continues to meet the needs of the thrombosis community.

\section{Local expansion}

Because of the very varied health care arrangements in different countries and the potential language barriers with our multinational participants, there has been a drive to regionalize and localize the program with the help of the global faculty and engaged experts in different countries, often themselves past alumni of a global INNOVATE meeting. This has made INNOVATE a truly global initiative, with programs now underway in Europe, North and South America, and the Asia-Pacific region (Figure S2). In addition, because many patients with NVAF and VTE are managed in the community setting, a new INNOVATE community care initiative aimed at primary and community care professionals was launched in 2016 with inaugural meetings in Manchester and Birmingham, UK, which I chaired.

Although differences between countries mean that a "one size fits all" approach is impossible, the localization of the program has demonstrated that common challenges exist, to which solutions based on the experiences of others, modified 
or otherwise, can be applied. Two examples of these regional and country initiatives are provided below.

\section{Russia}

In Russia, vascular surgeons tend to take the lead in DVT management in consultation with hematologists and clinical pharmacology specialists, and there is a stronger emphasis on interventional techniques for VTE treatment. As well as acute treatment, vascular surgeons also oversee the ambulatory/outpatient management of patients along with the patient's general practitioner (GP). The nurse-led model of thrombosis care used in London and Sheffield would not yet be accepted in Russia. Nevertheless, several prominent Russian physicians attended one of the global INNOVATE meetings in London, and subsequently I went to Russia to chair an INNOVATE kick-off meeting in Saint Petersburg in December 2014. The attendees were 14 "champions" for INNOVATE in Russia who wanted to set up regional INNOVATE meetings in seven centers of excellence across the country. Ultimately, it is hoped that this network will lead to the adoption of standard algorithms and protocols into national guidelines and other regulatory documents.

\section{Asia-Pacific region}

The management of VTE in Australia differs considerably between geographical locations and hospitals, leading to an inconsistent standard of diagnosis, treatment, and management of patients across the country. There are also different funding models in different states and a lack of guidance for GPs and others treating patients in the community, as well as the problem in rural communities of geographical isolation from core services. Owing to differences in the organization of services, Bayer Australia worked with an expert group from the Australian Society of Thrombosis and Haemostasis (ASTH) to set up an agenda for an initial pan-Australian INNOVATE meeting comprising a multidisciplinary faculty and participant group. Dr Rhona Maclean, co-chair of the Sheffield meeting, was invited to provide an overview of the Sheffield VTE pathway and of INNOVATE more broadly. Challenges within the Australian delivery of VTE services and specific case studies relating to the NOACs were discussed. The response to the meeting was overwhelmingly positive, and further meetings are planned. The ASTH intends to use INNOVATE to drive the development of standard care pathways. A similar model was used in other parts of the AsiaPacific region, with a multi-country meeting taking place in Singapore, chaired by me and Dr David Kiely, who co-chairs meetings in Sheffield. Both of these meetings highlighted a great diversity in practice but at the same time common areas from which learnings can be drawn. The next stage is to bring INNOVATE to the local level in these countries.

\section{Implementing learnings from INNOVATE in local VTE protocols}

The practical benefits of INNOVATE have been demonstrated by the creation and strengthening of existing systems for VTE patient management, driven by participants who have attended meetings. Two examples are presented below.

\section{Central Alberta Anticoagulation Clinic, Red Deer, Alberta, Canada}

Vanessa Higgins-Nogareda is a specialist anticoagulation nurse working in the Central Alberta Anticoagulation Clinic. The service has traditionally been a warfarin/low-molecularweight heparin monitoring clinic for all patients with an indication for anticoagulation and covers a wide geographical area. She attended the INNOVATE meeting in Hamilton in late 2014 with the aim of gaining knowledge about the NOACs and implementing protocols for managing patients receiving these agents who attend her clinic. Using information about the safety and efficacy of NOACs, contraindications and advice on patient counseling, as well as example materials from Hamilton and other centers shared at the meeting, Alberta-specific checklists and protocols were created. These cover questions addressing which patients are eligible and appropriate for switching from warfarin to a NOAC, how to discuss therapy with patients, what information to pass on to other health care professionals, how to provide follow-up, and channels of communication. Additionally, the clinic has supported the wider education of health care professionals in Alberta around NOACs through teaching sessions for hospital multidisciplinary teams (including surgery, anesthesiology, pre-admission services, emergency department staff, intensive care, cardiology, and internal medicine physicians) and GP office lunch meetings.

\section{Blackburn and Darwen National Health Service Trust, UK}

Irfan Zafar, a GP based in the east Lancashire local authority region of Blackburn, attended an INNOVATE meeting in London in late 2014. Historically, all patients in his region presenting with suspected DVT required either referral or admission to hospital for diagnosis and initiation of treatment, after which they were often required to attend a specialist 
clinic in a different town. A review of the service found that patient pathways were complicated and involved several levels of care, creating an inefficient, costly, and disjointed service. For this reason, and to comply with UK guidelines on VTE treatment, an upgrade to the service was proposed. The aims of the new service were to improve and simplify the care of patients with suspected DVT in a primary care setting through accurate and early diagnosis; to reduce inappropriate referral to secondary care; and to provide clear and consistent pathways with high standards of care. Electronic and telephone-based referral services were rolled out to speed up the process of diagnosis and treatment, benefiting both patients and GPs, and patients could now receive a same-day full assessment at one single primary care site. In the first 9 months of a 1-year pilot of this service, 238 patients with suspected DVT were assessed within 24 hours ( $\sim 60 \%$ were seen within 4 hours of referral), and 56 were diagnosed with DVT, of whom 48 were managed effectively using the new community pathway. Only $4 \%$ of patients required referral to secondary care (Irfan Zafar, unpublished data).

\section{How INNOVATE is run: roles and responsibilities}

Ideally, an INNOVATE meeting takes place at an existing center of excellence with the faculty mostly or entirely composed of multidisciplinary health care professionals who work together to manage patients with VTE. However, the examples above show that this does not have to be the case in regions or countries where such a center does not exist. The proposed chair and other senior faculty members should have attended a previous INNOVATE meeting so that they have experience of and can better understand the concept and format, and the agenda should generally be based around the hospital's established patient pathway (or an "ideal" pathway if one does not exist). Around 25 participants are invited, each completing a pre-meeting questionnaire to help the faculty understand their current level of experience and what objectives they have for attending the meeting. After the meeting, participants are asked to complete an evaluation questionnaire to guide the faculty in improving future meetings. The feedback from delegates has been consistently positive, but many useful suggestions for updates and improvements have been taken on board. The faculty is encouraged to maintain direct contact with the attendees to provide support should they have further questions and/or require advice as they implement new pathways and protocols in their institutions.

Bayer sponsors the INNOVATE program through a series of educational grants, maintaining a hands-off and compliant role in funding the program. Control of the meeting content rests entirely with the chairs and faculty, who develop and refine the program at each center with the objective of sharing best practice in patient management, rather than focusing on any particular drug. Potential participants are identified and invited by Bayer local staff, and also through recommendations of peers and previous attendees and the faculty themselves. Professional medical communications and logistics agencies assist in running the meetings, funded by the sponsor. Their role includes the development and adaptation of a standard series of materials (including, but not limited to, agendas, signage, invitations, evaluation forms, and slides) carrying the INNOVATE logo. Although these can be adapted for local use, the consistent format of these materials ensures that, whatever the country and language, INNOVATE remains recognized as a truly global network of thrombosis excellence.

\section{Conclusion}

The INNOVATE program is a growing worldwide initiative that showcases best practice in patient care in those requiring anticoagulation for thromboembolic disorders. INNOVATE seeks to provide a model for best practice in the non-promotional support of medical education by the pharmaceutical industry, the responsible use of new drugs and their integration into existing and new patient pathways. The ultimate aim is that every patient receives the benefit of evidence-based clinical practice.

\section{Acknowledgments}

I acknowledge my fellow INNOVATE chairs Dr Rhona Maclean and Dr David Kiely (Sheffield, UK), Dr Stavros Konstantinides (Mainz, Germany), Dr Jeffrey Weitz and Dr John Eikelboom (Hamilton, ON, Canada), who have reviewed and contributed to this article, as well as the delegates whose experiences are cited in this article Vanessa Higgins-Nogareda (Red Deer, AB, Canada) and Dr Irfan Zafar (Blackburn, UK). I also acknowledge Sarah Atkinson, who provided editorial support with funding from Bayer AG.

\section{Disclosure}

RP received speaker's bureau fees, honoraria, and funding for presentation materials from Bayer.

\section{References}

1. Anderson M. Joint working can bring benefits to all parties involved. 2010. Available from: http://www.guidelinesinpractice.co.uk/GinP. co.uk/mar_10_anderson_jw_mar10. Accessed October 18, 2016. 
2. Association of the British Pharmaceutical Industry (ABPI). Code of Practice for the Pharmaceutical Industry; 2016. London: Prescription Medicines Code of Practice Authority; 2016. Available from: http:// www.abpi.org.uk/our-work/library/guidelines/Documents/code_of_ practice_2016.pdf. Accessed June 3, 2016.

3. Raskob GE, Angchaisuksiri P, Blanco AN, et al. Thrombosis: a major contributor to global disease burden. Arterioscler Thromb Vasc Biol. 2014;34(11):2363-2371.

4. Bayer Pharma AG. Xarelto ${ }^{\circledR}$ (rivaroxaban) Summary of product characteristics; 2016. Available from: http://www.ema.europa.eu/docs/en_GB/ document_library/EPAR_-_Product_Information/human/000944/ WC500057108.pdf. Accessed October 18, 2016

5. Boehringer Ingelheim International $\mathrm{GmbH}$. Pradaxa ${ }^{\circledR}$ (dabigatran etexilate) Summary of product characteristics; 2016. Available from: http://www.ema.europa.eu/docs/en_GB/document_library/EPAR_Product_Information/human/000829/WC500041059.pdf. Accessed September 21, 2016.
6. Bristol-Myers Squibb, Pfizer. Eliquis ${ }^{\circledR}$ (apixaban) Summary of product characteristics; 2016. Available from: http://www.ema.europa. eu/docs/en_GB/document_library/EPAR_-_Product_Information/ human/002148/WC500107728.pdf. Accessed September 21, 2016.

7. Daiichi Sankyo Europe GmbH. Lixiana ${ }^{\circledR}$ (edoxaban) Summary of product characteristics; 2016. Available from: http://www.ema.europa. eu/docs/en_GB/document_library/EPAR_-_Product_Information/ human/002629/WC500189045.pdf. Accessed October 18, 2016.

8. Heidbuchel H, Verhamme P, Alings M, et al. Updated European Heart Rhythm Association practical guide on the use of non-vitamin K antagonist anticoagulants in patients with non-valvular atrial fibrillation. Europace. 2015;17(10):1467-1507.

9. Heidbuchel H, Berti D, Campos M, et al. Implementation of non-vitamin $\mathrm{K}$ antagonist oral anticoagulants in daily practice: the need for comprehensive education for professionals and patients. Thromb J. 2015;13:22.

10. Ferreira M, Barsam S, Patel JP, et al. Heavy menstrual bleeding on rivaroxaban. Br J Haematol. 2016;173(2):314-315. 


\section{Supplementary materials}

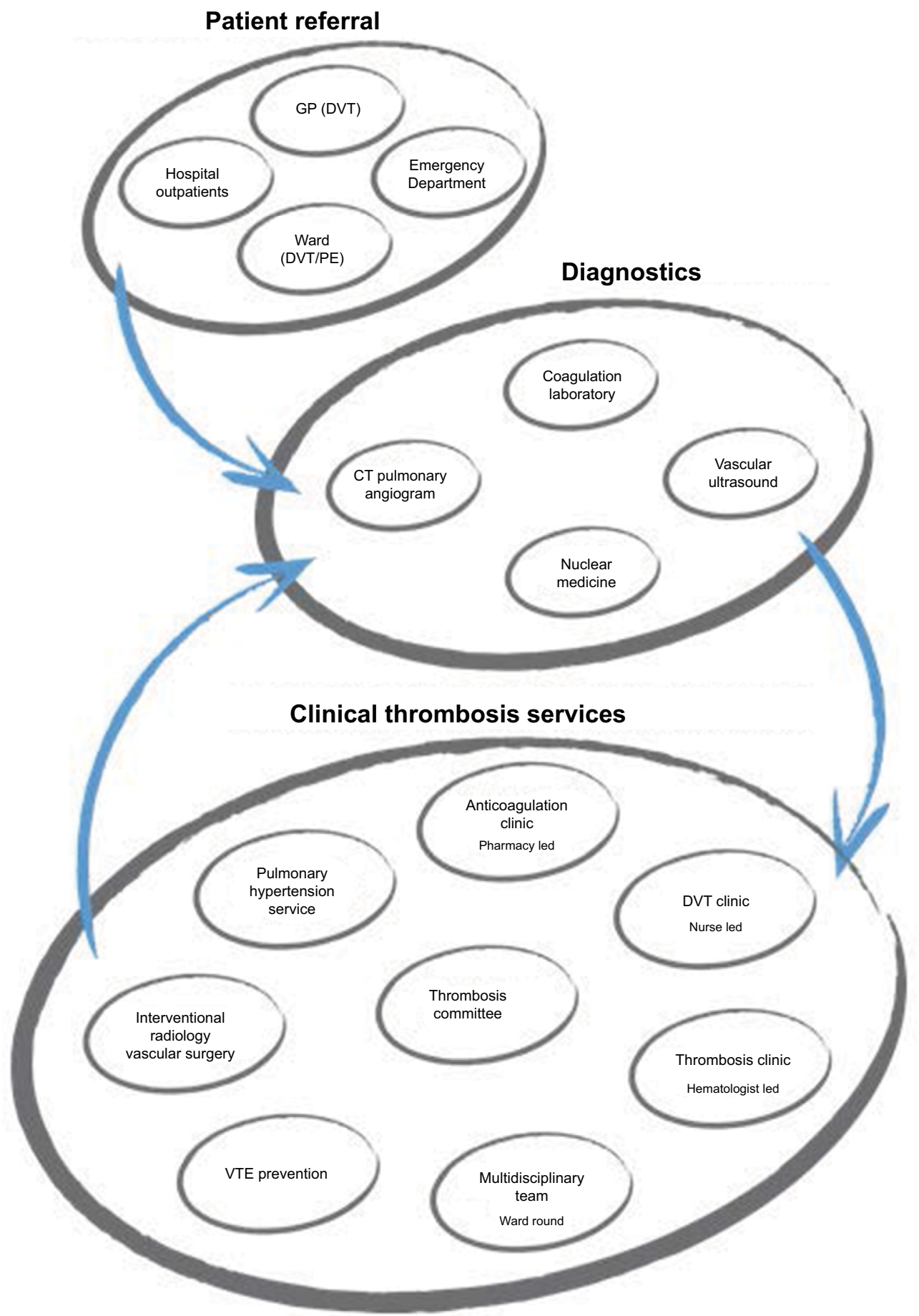

Figure SI The King's College Hospital patient pathway for VTE.

Abbreviations: CT, computed tomography; DVT, deep vein thrombosis; GP, general practitioner; PE, pulmonary embolism; VTE, venous thromboembolism. 


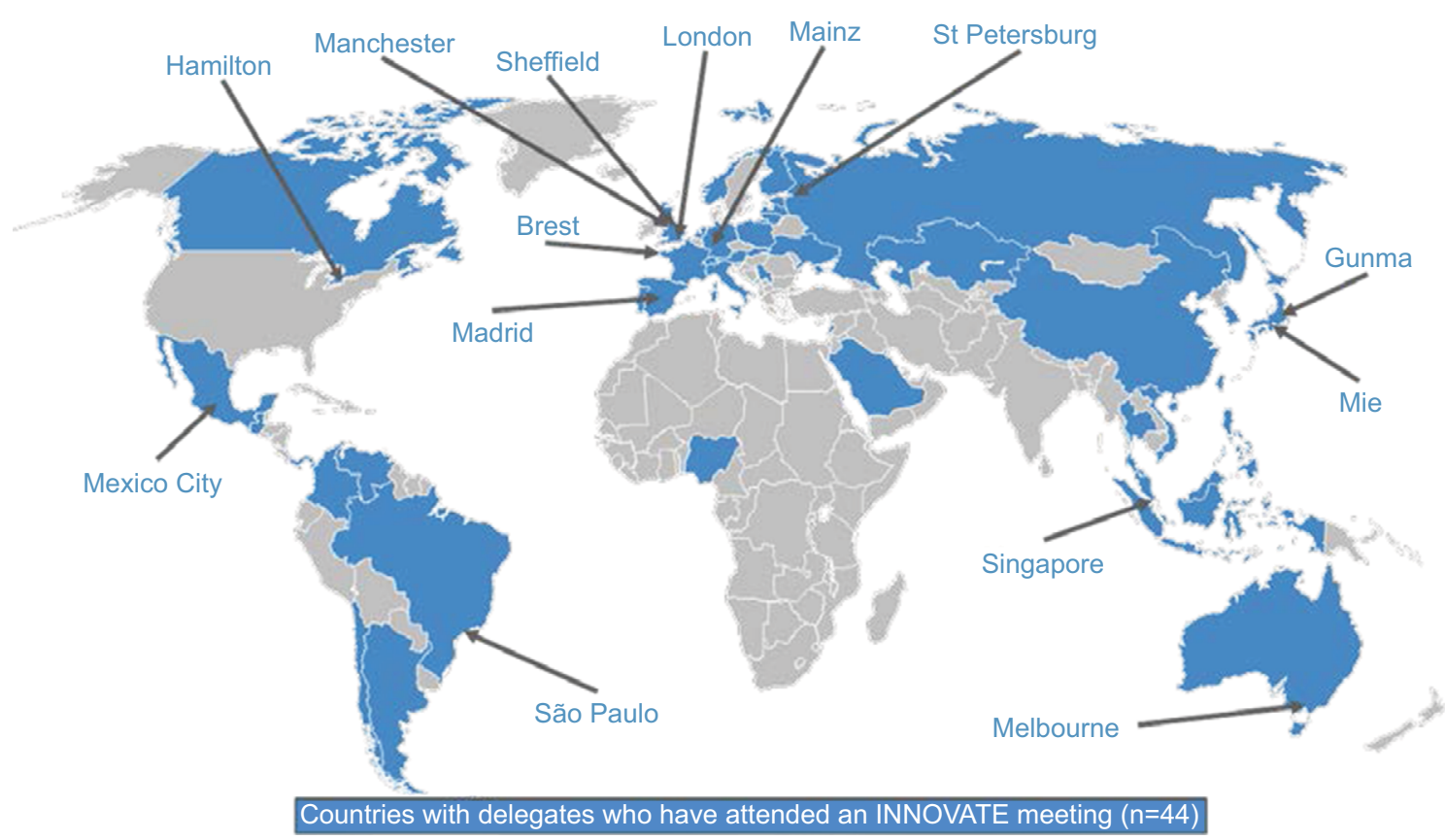

Figure S2 INNOVATE program: global reach to date. INNOVATE meetings have been held in the cities indicated. The blue shading represents the countries ( $=44$ ) with delegates who have attended an INNOVATE meeting.

Abbreviation: INNOVATE, INternational Network fOr Venous and Arterial Thrombosis Excellence in practice.

\section{Publish your work in this journal}

Advances in Medical Education and Practice is an international, peerreviewed, open access journal that aims to present and publish research on Medical Education covering medical, dental, nursing and allied health care professional education. The journal covers undergraduate education, postgraduate training and continuing medical education including emerging trends and innovative models linking education, research, and health care services. The manuscript management system is completely online and includes a very quick and fair peer-review system. Visit http://www.dovepress.com/testimonials.php to read real quotes from published authors.

Submit your manuscript here: http://www.dovepress.com/advances-in-medical-education-and-practice-journal 